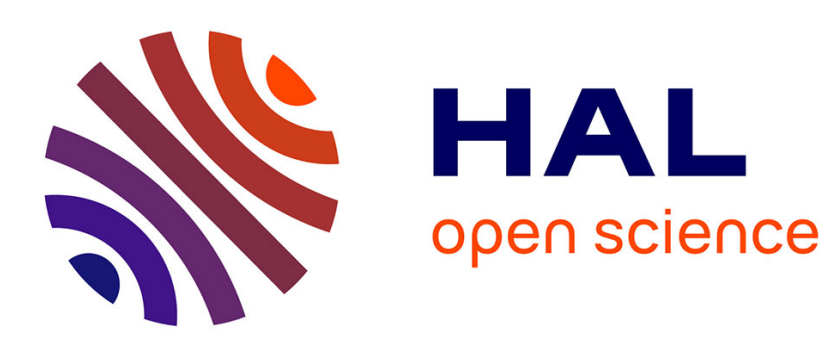

\title{
Port de combinaison et depense energetique lors d'un enchaînement natation-cyclisme
}

\author{
Anne Delextrat, Thierry Bernard, Christophe Hausswirth, Fabrice \\ Vercruyssen, Jeanick Brisswalter
}

\section{To cite this version:}

Anne Delextrat, Thierry Bernard, Christophe Hausswirth, Fabrice Vercruyssen, Jeanick Brisswalter. Port de combinaison et depense energetique lors d'un enchaînement natation-cyclisme. Canadian Journal of Applied Physiology, 2003, 28 (3), pp.356-369. 10.1139/h03-026 . hal-01823693

\section{HAL Id: hal-01823693 https://hal-insep.archives-ouvertes.fr/hal-01823693}

Submitted on 26 Jun 2018

HAL is a multi-disciplinary open access archive for the deposit and dissemination of scientific research documents, whether they are published or not. The documents may come from teaching and research institutions in France or abroad, or from public or private research centers.
L'archive ouverte pluridisciplinaire HAL, est destinée au dépôt et à la diffusion de documents scientifiques de niveau recherche, publiés ou non, émanant des établissements d'enseignement et de recherche français ou étrangers, des laboratoires publics ou privés. 


\title{
Port de combinaison et depense energetique lors d'un enchaînement natation-cyclisme
}

\author{
Anne Delextrat ${ }^{1} . z$, Thierry Bernard1, Christophe Hausswirth ${ }^{2}$, \\ Fabrice Vercruyssen1, et Jeanick Brisswalter ${ }^{1}$
}

Mots-clés: triathlon, coût énergétique, rendement mécanique, locomotion Key words: triathlon, energy cost, cycling efficiency, locomotion

\section{Résumé/Abstract}

L'objectif de cette étude était d'analyser l'influence du port de combinaison en natation sur la dépense énergétique en cyclisme. Neuf triathlètes entraînés ontparticipé à trois épreuves sous-maximales. Lors de la première épreuve (NSC), les sujets nageaient $750 \mathrm{~m}$ sans combinaison à allure de compétition, suivis de 10 min de cyclisme à une intensité correspondant au seuil ventilatoire $+5 \%$. Les deux autres épreuves étaient composées du même exercice sur ergocycle, précédé soit de $750 \mathrm{~m}$ de natation avec combinaison (NAC), soit d'un échauffement sur ergocycle ( Ctrl). Les résultats montrent que la natation avec combinaison se traduit par des valeurs inférieures de fréquence de rotation (-14\%), f réquence cardiaque $(-11 \%)$, et lactatémie $(-47 \%, p<0.05)$. Par ailleurs, le rendement mécanique en cyclisme est significativement supérieur après la condition NAC comparativement à la condition NSC (différence de $12,1 \%, p<.05$ ). La diminution de l'intensité de la natation avec combinaison suggère l'importance des conditions de la partie natation lors $d^{\prime}$ un triathlon sprint.

The aim of this study was to investigate the effects of swimming with a wetsuit on energy expenditure during subsequent cycling. Nine well-trained triathletes underwent three

${ }^{1}$ Laboratoire Ergonomie Sportive et Performance, EA 3162, Université de ToulonVar, Avenue de l'Université, BP 132, 83957 LaGarde Cedex, France; ${ }^{2}$ Laboratoire de Biomécanique et de Physiologie, INSEP, Avenue du Tremblay, Paris, France. 
submaximal trials. Thefirst trial (SC) consisted of a 750-m swim realised at a competition pace, followed by a JO-min cycling exercise at a power output corresponding to the ventilatory threshold $+5 \%$. The two other trials were composed of the same cycling exercise, preceded either by a 750-m swim with a wetsuit (WSC) or by a cycling warm-up (Ctrl). The main results are that the WSC trial was characterised by significantly Lower swimming cadence (-14\%), heart rate (-11\%), and lactate values (-47\%) compared to the SC trial, $p$ $<0.05$. Moreover, cycling efficiency was significantly higher in the WSC trial compared to the SC trial $(12.1 \%$ difference, $p<0.05)$. The Lower relative intensity observed during swimming with a wetsuit suggest the relative importance of swimming condition on the total peiformance in a sprint triathlon.

\section{Introduction}

Le triathlon est une activité de longue durée caractérisée par la réalisation successive d'épreuves de natation, de cyclisme, et de course à pied. Plusieurs types d'épreuves de triathlon se déroulent en compétition allant du triathlon sprint (750 $\mathrm{m}$ de natation, $20 \mathrm{~km}$ de cyclisme, $5 \mathrm{~km}$ de course à pied) au triathlon de type Ironman (4 km de natation, $180 \mathrm{~km}$ de cyclisme, $42 \mathrm{~km}$ de course à pied). La durée relative de chacune des trois disciplines varie respectivement entre le triathlon sprint et le triathlon de type Ironman de $18 \%$ à $10 \%$ pour la natation, $52 \%$ à $56 \%$ pour le cyclisme, et $30 \%$ à $44 \%$ pour la course à pied (Dengel et al., 1989; Hausswirth et al., 1999). Dans ce cadre, des corrélations significatives ont été observées entre la performance totale en triathlon et les temps réalisés lors des épreuves de cyclisme et de course à pied, alors que la performance totale ne semble pas être influencée par le temps de la partie natation (Dengel et al., 1989; Schabort et al., 2000).

Aussi, la plupart des études effectuées dans une perspective d'optimisation de la performance en triathlon se sont intéressées aux caractéristiques des épreuves de cyclisme et de course à pied du triathlon ou aux effets de la transition cyclismecourse à pied sur la performance en course à pied alors qu' à l'inverse, peu d'études ont abordé !'effet de l'épreuve de natation sur la performance cycliste (Laursen et al., 2000; Millet et Vleck, 2000). Néanmoins, certains travaux suggèrent qu'indépendamment du temps réalisé lors de l'épreuve de natation, la dépense énergétique liée à la nage peut affecter d'une part l'adaptation physiologique lors de la partie cyclisme et d'autre part, la performance totale en triathlon (Farber et al., 1991; Laursen et al., 2000; Margaritis, 1996).

Comme dans la plupart des activités de longue durée, la performance en triathlon dépend principalement de la capacité du sujet à maintenir une vitesse maximale de déplacement tout en réduisant la quantité d'énergie métabolique dépensée par unité de distance parcourue (Hausswirth et Lehénaff, 2001 ; O'Toole et Douglas, 1995). Des études récentes ont mis en évidence l'importance du niveau de sollicitation physiologique lors de la partie cyclisme sur la dépense énergétique et/ou la performance en course à pied. Par exemple, Hausswirth et al. (1999) ont observé que l'utilisation du drafting (déplacement dans l'aspiration d'un groupe) en cyclisme, qui se traduisait par une diminution significative de la dépense énergétique en cyclisme (-14\%), permettait d'augmenter la vitesse de déplacement de $4,1 \%$ pendant la course à pied d'un triathlon sprint. Ce résultat suggère que le 
niveau de dépense énergétique lors de la partie cyclisme peut être un facteur limitant la performance en triathlon.

Dans ce cadre, des valeurs de lactatémie sanguine élevées ont été observées après l'épreuve de natation qui mettent en évidence le rôle de cet exercice préalable sur l'état physiologique de l'athlète au début de l'épreuve de cyclisme (Farber et al., 1991). Deux questions peuvent alors être posées, l'une concernant l'influence de l'exercice préalable en natation sur l'adaptation physiologique lors des épreuves suivantes du triathlon, et la seconde concernant l'influence des conditions dans lesquelles la partie natation est réalisée sur une éventuelle modification du niveau de sollicitation physiologique lors de l'épreuve de cyclisme.

La plupart des travaux scientifiques menés en natation montrent que le niveau de dépense énergétiq ue dans cette discipline dépend en grande partie des résistances hydrodynamiques à l'avancement (Chatard et Millet, 1996; Lavoie et Montpetit, 1986). Deux moyens sont classiquement évoqués dans la littérature pour diminuer ces résistances: d'une part, l'utilisation du drafting, et d'autre part, le port d'une combinaison en néoprène. Lorsque le drafting est utilisé, des diminutions significatives de $\mathrm{VO}_{2}(-10 \%)$ et de la lactatémie (de -11 à $\left.-30 \%\right)$ ont été relevées dans la littérature pour des épreuves de 400 ou 500 m (Basset et al., 1991 ; Chollet et al., 2000). De même, il existe une diminution significative du coût énergétique des triathlètes lors d'un exercice avec port de combinaison comparé à un exercice sans combinaison (de 16,2\% à 33,6\% selon le type de combinaison, Trappe et al., 1996). Ce moindre coût semble être lié à une amélioration de la flottabilité des sujets, notamment au niveau des membres inférieurs, qui permet d'adopter une position plus horizontale dans l'eau, réduisant ainsi l'aire frontale présentée à l'avancement (Chatard et Millet, 1996; Chartard et al., 1995; Toussaint et al., 1989).

Dans une perspective d'optimisation de la performance en triathlon, nous émettons l'hypothèse selon laquelle le port d'une combinaison, en réduisant les résistances hydrodynamiques et par conséquent le niveau de dépense énergétique en natation, permet d'améliorer l'adaptation physiologique lors de la partie cyclisme. L'objectif de ce travail est alors d'étudier l'influence du port de combinaison en natation sur la dépense énergétique d'un exercice de cyclisme réalisé à la suite d'un enchaînement natation-cyclisme similaire à celui d'un triathlon.

\section{Méthodes}

SUJETS

Neuf sujets triathlètes de niveau régional à national ont participé à cette étude. Leurs caractéristiques sont exprimées comme la moyenne \pm écart-type (âge: 27 \pm 5 ans; taille: $179 \pm 7 \mathrm{~cm}$; poids: $71 \pm 9 \mathrm{~kg}$; masse grasse: $11 \pm 3 \%$ ). Tous les sujets étaient habitués aux épreuves sur ergocycle en laboratoire. Leur volume d'entraînement hebdomadaire comprenait en moyenne $5,3 \mathrm{~km}$ en natation, $95 \mathrm{~km}$ en cyclisme, et $32 \mathrm{~km}$ en course à pied, ce qui représentait environ $106 \mathrm{~min}, 196$ min, et $137 \mathrm{~min}$, respectivement, dans ces trois disciplines. Cette étude a été agréée par le comité d'éthique pour la protection des individus (Saint-Germain en Laye, France), et tous les sujets ont rempli un consentement écrit après avoir été informés en détail des procédures de l'expérimentation. 


\section{PROTOCOLE EXPERIMENTAL}

Chaque sujet a participé à quatre sessions expérimentales séparées les unes des autres d'au moins $48 \mathrm{~h}$. La première session correspondait à un test triangulaire (progressif maximal) de détermination de la consommation maximale d'oxygène $\left(\mathrm{VO}_{2} \max \right)$ et de la puissance atteinte à $\mathrm{VO}_{2} \max$ (PMA). Les trois autres sessions correspondaient à des tests rectangulaires sous-maximaux composés de deux situations d'enchaînement natation-cyclisme (avec et sans combinaison) et d' une situation contrôle de cyclisme isolé.

Session 1: Evaluation de $\mathrm{VO}_{2}$ max. Après un échauffement de 6 min à $10 \mathrm{O}$ $\mathrm{W}$, l'intensité mécanique était augmentée de $30 \mathrm{~W}$ par minute, jusqu'à ce que le sujet ne puisse plus maintenir la puissance imposée. Les critères d'atteinte de $\mathrm{VO}_{2}$ max étaient les suivants: un plateau de $\mathrm{VO}_{2}$ malgré l'augmentation de la puissance, une FC supérieure à $90 \%$ de la FCmax théorique, et un quotient respiratoire (QR) supérieur à 1,15 (Howley et al., 1995). A partir des valeurs de débit ventilatoire $(\mathrm{VE})$, de consommation d'oxygène $\left(\mathrm{VO}_{2}\right)$, et de production de dioxyde de carbone $\left(\mathrm{VCO}_{2}\right)$, le seuil ventilatoire $(\mathrm{SV})$ était déterminé selon la méthode décrite par Wasserman (1973).

Sessions 2, 3, et 4: Tests sous-maximaux. Chaque triathlète a réalisé trois épreu ves sous-maximales. La première épreuve était toujours constituée de l'enchaînement natation sans combinaison-cyclisme (NSC), tandis que les deux autres épreuves étaient présentées dans un ordre aléatoire (figure 1).

L'épreuve NSC consistait à nager $750 \mathrm{~m}$ à l'allure de compétition, puis à réaliser une épreuve de 10 min sur ergocycle à une intensité correspondant à $\mathrm{SV}$ $+5 \%$. Cette intensité a été choisie parce qu'elle est représentative de l' allure adoptée par nos sujets en compétition lors de triathlons sprints. Par ailleurs, elle est comparable aux intensités utilisées lors de travaux récents étudiant la transition cyclismecourse à pied chez des triathlètes entraînés (e.g., Hausswirth et al., 1997; Hue et al., 1998). Pendant la seconde épreuve d'enchaînement avec combinaison (NAC), les sujets nageaient $750 \mathrm{~m}$ avec leur propre combinaison de triathlon à la même allure que !'épreuve NSC, puis enchaînaient sur l'ergocycle à la même intensité qu'en situation NSC. Le même type de combinaison était utilisé par tous les sujets (combinaison intégrale Aquaman ${ }^{\circledR}$, modèle Pulsar 2000, épaisseur épaules: 1,5 $\mathrm{mm}$; tronc: $4,5 \mathrm{~mm}$; jambes: $1,5 \mathrm{~mm}$; bras: $1,5 \mathrm{~mm}$ ). Afin de reproduire la vitesse de nage adoptée dans la situation NSC, les sujets étaient informés visuellement de leurs performances à chaque $50 \mathrm{~m}$.

La troisième épreuve (condition contrôle, Ctrl) était uniquement composée de l'exercice de 10 min sur ergocycle précédé d'un échauffement à une puissance de $30 \%$ de PMA pendant la même durée que l'épreuve de natation sans combinaison. Toutes les épreuves sur ergocycle étaient réalisées à la cadence spontanément adoptée par les sujets (CL, rév·min- ${ }^{1}$ ), qui était enregistrée tout au long du test.

\section{PARAMÈTRES MESURES}

Epreuves de natation. Les sujets ont réalisé deux épreuves de natation correspondant aux conditions avec ou sans le port d'une combinaison intégrale. Ces épreuves se déroulaient dans le bassin extérieur de 50 mètres de la piscine de Hyères (Var, France) où la température moyenne de l'eau était de $28{ }^{\circ} \mathrm{C}$ pour une 


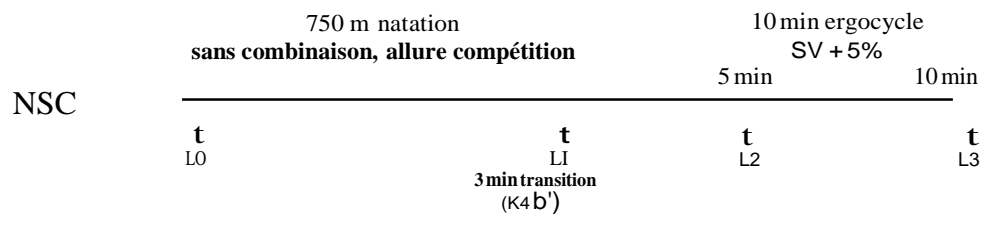

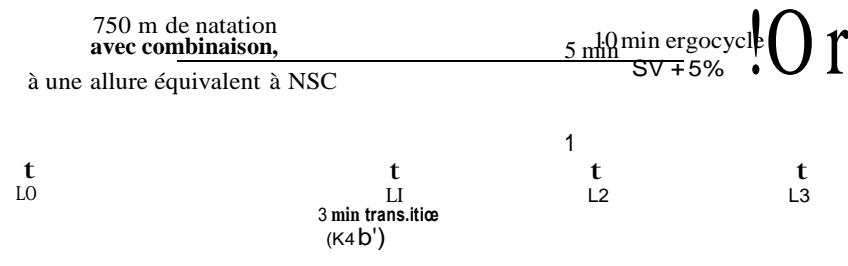

NAC $3 \min \operatorname{trans}$
$\left(\mathrm{K} 4 \mathrm{~b}^{\prime}\right)$ ergocycle de durée équivalente à NSC $(30 \%$ de PMA

$$
\begin{gathered}
10 \mathrm{~min} \text { ergocycle } \\
\mathrm{SV}+5 \%
\end{gathered}
$$

CTRL

$\begin{array}{cccc}\mathbf{t} & \mathbf{t} & \mathbf{t} & \mathbf{t} \\ \mathrm{LO} & \mathrm{L} & \mathrm{L} & \mathrm{L} \\ & \left(\mathrm{K} 4 \mathrm{~b}^{\prime}\right) & & \end{array}$

Figure 1. Protocole de l'étude. L:prélèvement sanguin, $\mathrm{K} 4 \mathrm{~b}^{2}$ installation du cosmed $\mathrm{K} 4 \mathrm{~b}^{2}$

température moyenne de l'air de $14,3^{\circ} \mathrm{C}$. Durant les deux épreuves de natation, la performance finale ainsi que les temps réalisés à chaque $50 \mathrm{~m}$ étaient relevés. La fréquence de rotation des bras, quantifiée par le nombre de cycles de bras complets réalisés par min, était mesurée sur une distance de $25 \mathrm{~m}$ située au milieu du bassin.

Ergocycle. Toutes les épreuves de cyclisme se déroulaient sur un ergocycle à résistance électromagnétique (SRM Jülich, Welldorf, Allemagne) dans une pièce proche du bassin afin que la durée des transitions natation-cyclisme puisse être standardisée ( $3 \mathrm{~min}$ ). L'ergocycle était équipé avec les propres pédales des triathlètes et pouvait s'ajuster précisément à leurs caractéristiques anthropométriques grâce à un réglage horizontal et vertical de la selle et du cintre. Le mode de fonctionnement de l'ergocycle permettait la production d'une puissance constante indépendamment de la cadence de pédalage naturellement adoptée par les sujets.

Mesure de la lactatémie. Un échantillon de sang capillarisé était recueilli au lobe de l'oreille au repos (LO), immédiatement après l'épreuve de natation (L 1 ), à la 5ème et la 1Qème minute de cyclisme (L2 et L3). A partir de ces échantillons, une mesure de la lactatémie ([La-], mmol·L- ${ }^{1}$ ) était obtenue grâce à l'analyseur Lactate Pro, validé par Pyne et al. (2000). 
la consommation d'oxygène $\left(\mathrm{VO}_{2}\right)$, la fréquence cardiaque $(\mathrm{FC})$ ainsi que les paramètres respiratoires (débit ventilatoire: VE; fréquence respiratoire: FR) étaient enregistrés en continu par un système d'analyse télémétrique cycle à cycle de type Cosmed $\mathrm{K}_{4} \mathrm{~b}^{2}$ (Rome, Italie) validé par McLaughlin et al. (2001). Ces valeurs étaient recueillies puis moyennées sur une période de 2 minutes, respectivement, entre la 3ème et la 5ème minute et entre la Sème et la 1Oème minute.

Parallèlement, la FC était enregistrée pendant toute la durée des épreuves (natation et cyclisme) grâce à un cardiofréquencemètre de type Polar vantage (Finlande).

Dans cette étude, le rendement mécanique global du cyclisme (I'I, en \%) était calculé comme le rapport entre le travail mécanique accompli par min (i.e., watts convertis en $\mathrm{kJ} \cdot \mathrm{min}-1)$ et l'énergie métabolique dépensée par min $(\mathrm{kJ} \cdot \min -1$, Chavarren et Calbet, 1999). L'intensité des exercices sur ergocycle étant supérieure au seuil ventilatoire $(\mathrm{SV}+5 \%)$; l'énergie métabolique a été calculée à partir des équivalents énergétiques de l'oxygène (en fonction de la valeur de quotient respiratoire) et du lactate (63 J·kg-l.mM- $\left.{ }^{1}\right)$ (di Prampero, 1981).

\section{ANALYSE STATISTIQUE}

Pour chaque variable, la valeur moyenne et l'écart-type étaient calculés. L'influence du type d'exercice préalable (i.e., conditions NSC, NAC, et Ctrl) sur les différents indices physiologiques $\left(\mathrm{VO}_{2}\right.$, rendement, $\mathrm{FC}, \mathrm{VE}, \mathrm{FR}$, lactatémie et $\left.\mathrm{CL}\right)$ était analysée par une analyse de variance (ANOVA 2R) à mesures répétées (condition $\mathrm{x}$ période, logiciel statistica version 5.0). Puis une comparaison des variables entre les différentes conditions expérimentales était réalisée par un test post-hoc de type Newman-Keuls. Les différentes valeurs étaient rapportées à un seuil de signification de $p<0.05$.

\section{Résultats}

TEST TRIANGULAIRE (PROGRESSIF MAXIMAL)

Les principaux résultats obtenus lors de ce test sont présentés au tableau 1. Les valeurs de FCmax et de QRmax indiquent que les critères d'atteinte de $\mathrm{V}_{2}$ max

Tableau 1 Résultats du test triangulaire

\begin{tabular}{|c|c|c|c|c|c|}
\hline $\begin{array}{c}\mathrm{V}_{2} \max \\
\left(\mathrm{ml} \cdot \mathrm{min}^{-1} \cdot \mathrm{kg}^{1}{ }^{1}\right)\end{array}$ & $\begin{array}{l}\text { FCmax } \\
(\text { batt } \cdot \min -1)\end{array}$ & $\begin{array}{l}\text { PMA } \\
\text { (Watts) }\end{array}$ & $\begin{array}{l}\text { QR } \\
\max \end{array}$ & $\begin{array}{l}\text { Puissance à SV } \\
\text { (Watts) }\end{array}$ & $\begin{array}{c}\text { V02 à SV } \\
(\mathrm{ml} \cdot \mathrm{min}-1 . \mathrm{kg}-1)\end{array}$ \\
\hline 71,7 & 193 & 379,7 & 1,11 & 255,9 & 50,6 \\
\hline $\pm 6,1$ & \pm 9 & $\pm 39,0$ & $\pm 0,07$ & $\pm 24,8$ & $\pm 4,8$ \\
\hline
\end{tabular}


sont respectés. Par ailleurs, les valeurs de $\mathrm{VO}_{2} \mathrm{max}$ obtenues dans cette étude sont comparables à celles de triathlètes de même niveau (Brisswalter et al., 2000; Vercruyssen et al., 2001).

\section{EPREUVE PREALABLE DE NATATION}

Performance. Aucune différence significative n'est observée entre les performances moyennes des deux épreuves de natation (respectivement pour les épreuves NSC et NAC: $716 \pm 64 \mathrm{~s}$ vs. $713 \pm 62 \mathrm{~s}, p>0.05)$, qui étaient réalisées à une vitesse moyenne de $1,05 \mathrm{~m} \cdot \mathrm{s}^{-1}$. Par contre, la fréquence de rotation des bras est significativement inférieure dans la situation NAC par rapport à NSC tout au long de la course (diminution moyenne de $14 \%$, figure $2, p<0.05$ ). Aucune variation significative de la fréquence de rotation avec la durée de 1 'exercice n'est observée dans la situation NSC, alors que la situation NAC est caractérisée par une diminution significative de la fréquence de rotation avec la durée de l'exercice (figure $2, p<0.05$ ).

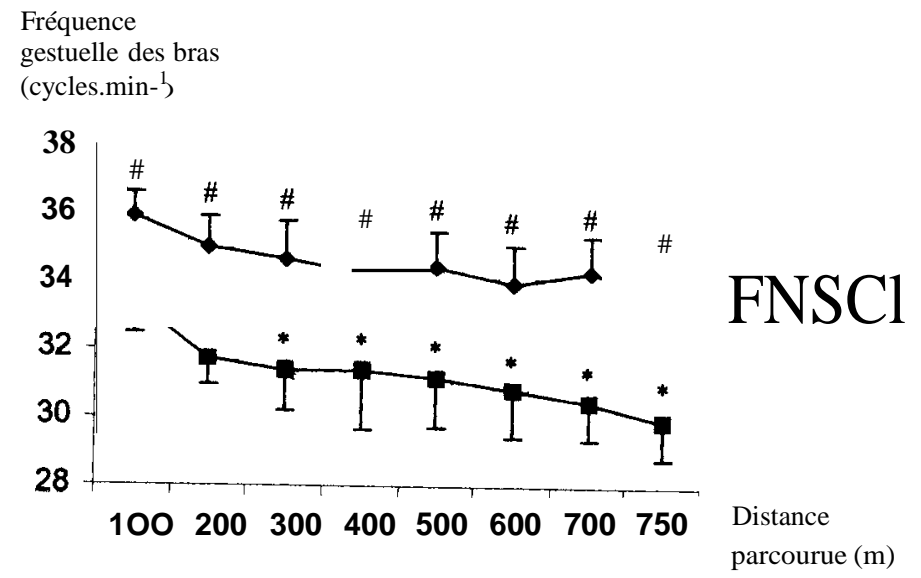

Figure 2. Evolution de la fréquence gestuelle des bras au cours des deux épreuves de natation (NAC et NSC). Significativement différent, $p<.05$ : *de la valeur mesurée aux $100 \mathrm{~m}$; \#de la condition NAC.

Indices physiologiques. L'évolution des valeurs de FC pendant les 10 dernières minutes des deux épreuves de natation est présentée à la figure 3. Les valeurs de FC relevées lors des 5 dernières minutes de natation et de [La-] mesurée immédiatement après la natation sont significativement inférieures dans la situation NAC par rapport à NSC (diminutions moyennes de $11 \%$ et $47 \%$, respectivement, pour la FC et [La-], figure $3, p<0.05$ ). Par ailleurs, ces valeurs sont significative-ment supérieures à celles mesurées immédiatement après l'échauffement 


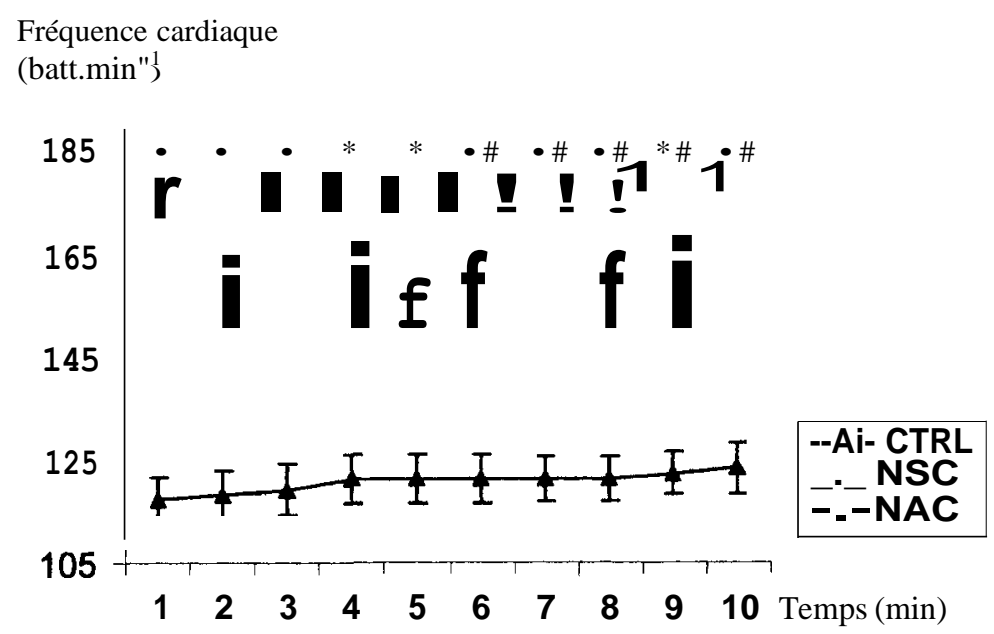

Figure 3. Evolution de la fréquence cardiaque au cours des 10 dernières minutes des deux épreuves de natation (NAC et NSC) et de l'échauffement sur ergocycle (Ctrl). Significativement différent, $p<.05: *$ de la condition Ctrl; \#de la condition NAC.

sur ergocycle (respectivement pour NSC, NAC, et Ctrl: [La-] $=9,1 \pm 2,4$ mmoJ-L-1 vs. $4,8 \pm 1,9$ mmoJ-L- 1 vs. $1,1 \pm 0,4$ mmol-L- $1, \mathrm{p}<0.01$; fréquence cardiaque $=$ $179 \pm 9$ batt $\cdot$ min- $^{-1}$ vs. $158 \pm 19$ batt $\cdot \min ^{-1}$ vs. $124 \pm 13$ batt $\left.\cdot \min ^{-1}, \mathrm{p}<0.01\right)$.

\section{EPREUVES SUR ERGOCYCLE}

L'influence du type d'exercice préalable sur les différents paramètres mesurés est présentée au tableau 2. Quelle que soit la condition, aucun effet de la période de mesure n'est observé. Les valeurs données représentent donc la valeur moyenne des deux périodes de recueil des paramètres (3-5 min et 8-10 min). Par ailleurs, aucune variation significative de la masse des sujets n'est mise en évidence entre le début et la fin des épreuves .

Effet de l' exercice préalable (NAC et NSC vs. Ctrl). L'analyse statistique révèle des valeurs de [La-] et de FC significativement supérieures lors des deux épreuves de natation comparée à l'épreuve $\mathrm{Ctrl}, p<0.05$. Par contre, seules les valeurs de V02' VE, et FR relevées à la suite de la situation NSC sont significativement supérieures à celles de la situation $\mathrm{Ctrl}, p<0.05$.

Effet du port de combinaison (NAC vs. NSC). L'analyse de variance indique un effet significatif du port de combinaison sur les paramètres cardiorespiratoires ainsi que sur les valeurs de lactatémie. Des diminutions significatives entre les conditions NSC et NAC sont observées pour $\mathrm{VO}_{2}(-4,5 \%)$, FC $(-3,3 \%)$, VE $(-14,4 \%)$, FR $(-15,6 \%)$, et [La-] (-25\%). Ainsi, le rendement mécanique du cyclisme est significativement pl us faible à la suite d'une épreuve de natation sans port de combinaison (différence de $12,1 \%$ entre NSC et NAC, figure $4, p<0.01$ ). 
Tableau 2 Effet du type d'exercice préalable sur les paramètres physiologiques et sur la cadence de pédalage libre au cours des trois épreuves sous-maximales sur ergocycle

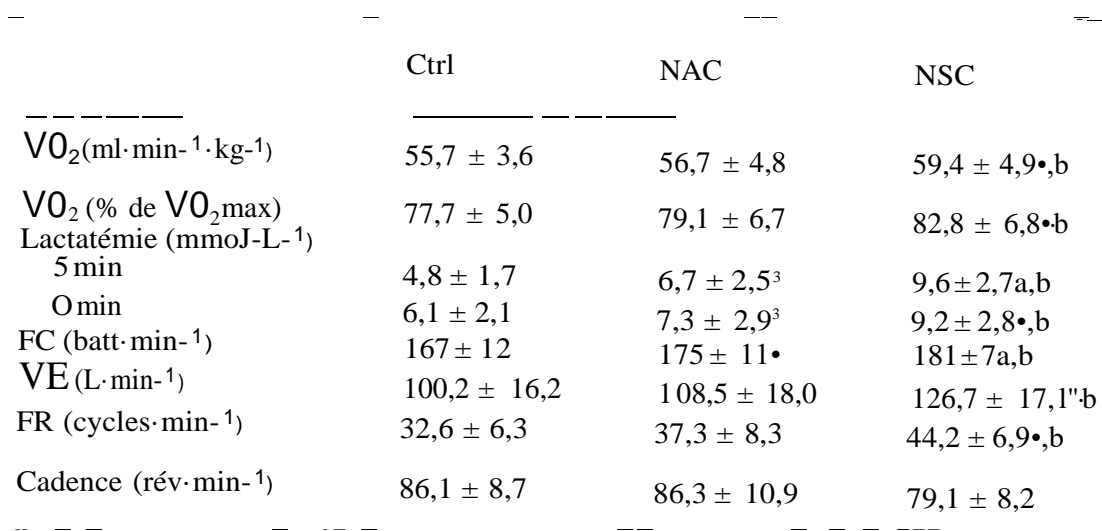

Significativement différent, $p<.05$ : a de la situation Ctrl; hde la situation NAC.

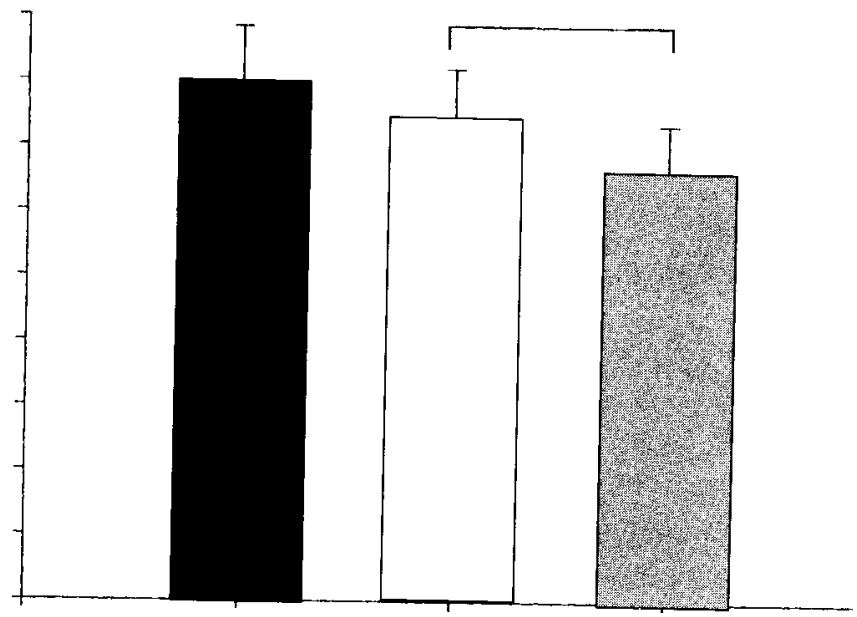

Figure 4. Effets du type d'exercice préalable sur le rendement mécanique du cyclisme (en \%). Significativement différent, $p<.05$ : a de la situation Ctrl; hde la situation NAC. 
Enfin, quelle que soit la condition, aucune variation significative de la cadence spontanée de pédalage n'a été observée dans cette étude, $p>0.05$.

\section{Discussion}

Le principal résultat de cette étude est l'effet significatif du niveau de sollicitation physiologique induit par une épreuve dont la vitesse était préalablement contrôlée sur l'adaptation physiologique en cyclisme. Dans ce cadre, pour un exercice de performance identique (i.e., même distance et même vitesse), le port de combinaison en natation se traduit par une moindre dépense énergétique lors de l'exercice subséquent de cyclisme.

\section{EFFET DU PORT DE COMBINAISON}

L'évolution des valeurs de FC au cours des deux épreuves de natation de cette étude (figure 3) montre des valeurs moyennes de FC qui correspondent pour NAC et NSC, respectivement, à $81 \%$ et $92 \%$ de FCmax mesurée en cyclisme, $p<0.05$. Dans notre étude, Je port de combinaison se traduit donc par une diminution significative de FC de $11 \%$ au cours d'un exercice de $750 \mathrm{~m}$ de natation. Ce résultat est comparable à ceux rapportés dans la revue de Chatard et Millet (1996), et supérieur aux résultats observés par Trappe et al. (1996) pour le même type de combinaison recouvrant l'intégralité du corps (diminution non significative de la FC de $5 \%$ pour une vitesse de nage de $1,1 \mathrm{~m} \cdot \mathrm{s}-1)$.

Le principal facteur évoqué dans la littérature pour expliquer la moindre dépense énergétique avec le port d'une combinaison est la diminution des résistances hydrodynamiques à l'avancement, respectivement, de 9 à $20 \%$ pour les résistances passives (Chatard et al., 1995) et de 12 à $16 \%$ pour les résistances actives (Toussaint et al., 1989). Les diminutions les plus importantes (-16\% et $-20 \%)$ sont observées pour des vitesses de nage relativement faibles $(1,1 \mathrm{~m} . \mathrm{s}-1)$ qui sont proches de celles relevées dans notre étude. Or, à ces vitesses, on observe classiquement une inflexion des membres inférieurs dans l'eau, qui contribue à augmenter l'aire frontale à l'avancement.

Nous suggérons donc que dans notre étude, le port de combinaison a provoqué une diminution maximale des résistances hydrodynamiques par rapport à l'épreuve de natation sans combinaison. Aucune mesure de ces résistances n'a été effectuée au cours de la présente étude, mais la variation de la fréquence gestuelle des bras au cours des deux épreuves de natation révèle des différences dans l'adaptation de l'individu à la nage. En effet, pour une vitesse de déplacement identique, la situation NSC se caractérise par une plus grande fréquence gestuelle et donc par un moindre travail mécanique (quantifié par la distance parcourue par cycle de bras) par rapport à la situation NAC.

D'autre part, des phénomènes de fatigue pendant la nage peuvent être mis en évidence dans la condition NSC. En effet, alors que la fréquence gestuelle diminue de façon significative avec la durée en NAC, indiquant que les forces propulsives développées sont de plus en plus importantes, la condition NSC est caractérisée par une stabilité de la fréquence gestuelle, caractérisant la diminution de la capacité des sujets à développer des forces propulsives (Craig et al., 1985). Nous pouvons 
donc suggérer que l'absence de port de combinaison se traduit par un niveau de dépense énergétique plus important au cours de 1 'épreuve de natation, et par l'apparition de phénomènes de fatigue en fin d'épreu ve qui peuvent affecter la performance lors de l'épreuve de cyclisme subséquente.

Dans notre étude, la comparaison des épreuves sur ergocycle montre que les valeurs de $\mathrm{VO}_{2}$ et [La-] sont significativement inférieures dans la condition NAC par rapport à la condition NSC ( $p<0.05$, tableau 2$)$. Ainsi, le rendement mécanique du cyclisme, déterminé à partir de ces deux paramètres, est significativement inférieur à la suite d'une épreuve de natation avec combinaison $(p<0.05$, figure

4). En cyclisme, pl usieurs facteurs sont classiquement évoqués pour expliquer les différences de rendement sur un exercice de courte durée. Parmi ceux-ci. les plus classiquement cités concernent les côtes de la bicyclette (induisant une position particul ière du sujet sur la bicyclette) et la cadence de pédalage (e.g., Gnehm et al., 1997; Hagan et al., 1992). Dans notre étude, le cycloergomètre SRM est calibré de 1 a même façon pour chaq ue condition expérimentale, afin de respecter les caractéristiques des bicyclettes de chaque coureur, et aucune variation significative de la cadence de pédalage n'est observée entre les différentes conditions expérimentales. Par ailleurs, la position des sujets sur 1 'ergocycle était précisément contrôlée afin d'éviter que des mouvements parasites dégradent le rendement mécanique.

Ainsi, l 'augmentation du coût énergétique dans la situation NSC ne peut pas être expliquée par ces deux facteurs. Aucune variation significative de $\mathrm{VO}_{2}$ avec la durée $\mathrm{n}$-aété mise en évidence au cours de l'épreuve sur ergocycle (respectivement pour NSC et NAC aux deux périodes: $\mathrm{VO}_{2}=58.6 \pm 4.5 \mathrm{vs} .60 .3 \pm 5.8 \mathrm{ml} \cdot \mathrm{min}^{-1} \cdot \mathrm{kg}^{-1}$, et $56.3 \pm 4.5$ vs. $57.2 \pm 5.2 \mathrm{ml} \cdot \mathrm{min}-1 . \mathrm{kg}-{ }^{1}, p>0.05$ ). Par conséquent, l'utilisation d'une plus grande fraction de $\mathrm{VO}_{2}$ max dans la situation NSC (respectivement pour NSC et NAC: $82.8 \pm 6.8 \%$ vs. $79.1 \pm 6.7 \%$ de $\left.\mathrm{VO}_{2} \max , p<0.05\right)$ s'explique essentiellement par !'augmentation de la dépense énergétique au cours de!'épreuve de natation induite par une moindre flottabilité de !'athlète et des phénomènes de fatigue dans les muscles actifs des membres inférieurs (Zamparo et al., 2000). Dans ce cadre, certains auteurs ont montré que la variation de l'intensité relative d'un exercice et/ou l'apparition de phénomènes de fatigue périphérique s'accompagnait d'une modification de l'importance ou de la nature du recrutement des fibres musculaires (Woledge, 1998).

Ainsi, il est classiquement indiqué que !'élévation de !'intensité relative d'un exercice se traduit par une augmentation du pourcentage de fibres de type II recrutées (Sargeant, 1994). Ces fibres musculaires sont caractérisées par un métabolisme à dominante glycolytique et une efficacité thermodynamique plus faible que les fibres musculaires de type 1(Coyle et al., 1992). Par ailleurs, Woledge (1998) indique qu'avec l'apparition de phénomènes de fatigue, les fibres qui sont initialement recrutées pour réaliser la tâche ne permettent plus de poursuivre le travail mécanique. Elles sont alors remplacées par d'autres types de fibres. Lors d'un exercice de pédalage à cadence libre et d'intensité sous maximale, les fibres de type 1 semblent être préférentiellement utilisées pour des cadences médianes (entre 60 et $90 \mathrm{rév} \cdot \mathrm{min}-{ }^{1}$, Barstow et al., 1996). Nous pouvons donc ainsi suggérer que 1 'élévation du niveau d'intensité et !'apparition de la fatigue dans les muscles des membres inférieurs se traduisent par un recrutement plus important de fibres musculaires de type II au 
début de !'exercice sur ergocycle dans la situation NSC par rapport à la situation NAC. Cette hypothèse expliquerait, pour une part, les valeurs plus importantes de $\mathrm{VO}_{2}$ et [La-] observées à la suite de l'épreuve sans combinaison.

\section{EFFET DU TYPE D"EXERCICE PRÉALABLE DE NATATION}

A notre connaissance, peu de travaux se sont intéressés aux effets d'un exercice préalable de natation sur la performance en cyclisme et les résultats semblent contradictoires. Récemment, Laursen et al. (2000) n'ont observé aucun effet d'une épreuve de $3000 \mathrm{~m}$ de natation sur la dépense énergétique en cyclisme. A l'inverse, d'autres auteurs ont montré une réduction significative du rendement mécanique du cyclisme à la suite de l'épreuve de natation d'un triathlon de courte distance (Hausswirth et al., 1999; Kreider et al., 1988). Par ses caractéristiques, l'épreuve de natation de notre étude se rapproche de celles d'Hausswirth et al. (1999) et de Kreider et al. (1988): distances de $750 \mathrm{~m}$ dans notre étude et de $750 \mathrm{~m}$ et $800 \mathrm{~m}$. respectivement, pour Hausswirth et al. et pour Kreider et al.

Lors de l'épreuve sans combinaison (NSC), nous confirmons les résultats précédemment décrits qui indiquent une diminution du rendement mécanique du cyclisme après une épreuve de natation. Par contre, notre étude ne révèle aucune différence significative entre les valeurs de $\mathrm{VO}_{2}$ et [La-] mesurées dans la condition contrôle ( $\mathrm{Ctrl}$ ) et la condition de nage avec combinaison (NAC) en début d'épreuve cyclisme (tableau 2). Seules les valeurs de [La-] de fin d'exercice de cyclisme diffèrent entre les deux épreuves. La contribution pl us importante du métabolisme anaérobie entre les conditions NAC et Ctrl pourrait être expliq uée par 1 'exercice préalable sur ergocycle qui met enjeu pendant une durée équivalente à la nage les muscles locomoteurs spécifiques de ce type d'effort. En effet, Bohnert et al. (1998) ont montré qu'une sollicitation préalable des membres inférieurs se traduisait par des valeurs plus faibles de [La-] et de $\mathrm{VO}_{2}$ lors d'un exercice sousmaximal de cyclisme, par rapport à une sollicitation préalable des membres supérieurs, $p<0.05$.

Bien que les mécanismes sous-jacents à cet effet restent mal définis, l'hypothèse principale suggère l'influence de facteurs hémodynamiques sur la vitesse et l'amplitude de la consommation d'oxygène et/ou sur l'acidose musculaire (Bohnert et al., 1998). Ces auteurs indiq uent que la réalisation d' un exercice préalable de même type (i.e., cyclisme-cyclisme) améliore la perfusion vasculaire dans les membres actifs au début du second exercice, augmentant ainsi la disponibilité en oxygène.

En conclusion, nos résultats supportent donc 1 'hypothèse évoquée par Margaritis (1996) selon laquelle le niveau métabolique induit par !'épreuve de natation peut avoir une influence non négligeable sur la performance en triathlon. Ces observations permettent également de mettre en évidence l'importance de l'intensité à laquelle l'épreuve de natation est réalisée sur la dépense énergétique de l'exercice de cyclisme. Par ailleurs, ce travail souligne l'intérêt du port d'une combinaison intégrale lors de la partie natation d'un triathlon sprint. Des travaux ultérieurs étudiant l'effet de la variation de !'intensité relative de nage sur la performance totale d'un triathlon seraient nécessaires afin de préciser l'influence de cette discipline sur la performance en triathlon. 


\section{Références}

Barstow, T.J ., Jones, A.M., Nguyen, P.H., and Casaburi, R. (1996). Influence of muscle fiber type and pedal frequency on oxygen uptake kinetics of heavy exercise. J. Appt. Physiol. 81: 1642-1650.

Basset, D.R., Flohr, J., Duey, W.J., Howley, E.T., and Pein, R.N. (1991). Metabolic responses to drafting during front crawl swimming. Med. Sei. Sports Exerc. 23: 744-747.

Bohnert, B., Ward, S.A., and Whipp, B.J. (1998). Effects of prior arm exercise on pulmonary gas exchange kinetics during high-intensity leg exercise in humans. Exp. Physiol. 83: $57-570$.

Brisswalter, J., Hausswirth, C., Smith, D., Vercruyssen, F., and Vallier, J.M. (2000). Energetically optimal cadence vs. freely-chosen cadence during cycling: Effect of exercise duration. lot. J. Sports Med. 21 : 60-64.

Chatard, J.C., and Millet, G. (1996). Effects of wetsuit use in swimming events. Sports Med. 22: 70-75.

Chatard, J.C, Senegas, X., Selles, M., Dréano, P., and Geyssant, A. (1995). Wet suit effect: A comparison between competitive swimmers and triathletes. Med. Sei. Sports Exerc. 27: $580-586$.

Chavarren, J., and Calbet, J.A.L. ( 1999). Cycling efficiency and pedalling frequency in road cyclists. Eur. J. Appt. Physiol. 80: 555-563.

Chollet, D., Hue. O., Auclair, F., Millet, G., and Chatard, J.C. (2000). The effects of drafting on stroking variations during swimming in elite male triathletes. Eur. J.Appl. Physiol. 82: 41 3-417.

Coyle, E.F., Sidossis, L.S., Horowitts, J.F., and Beltz, J.D. ( 1992). Cycling efficiency is related to the percentage of type 1 muscle fibres. Med. Sei. Sports Exerc. 24: 780-782.

Craig, A.B., Skehan, P.L., Pawelczyk, J.A., and Boomer, W.L. (1985). Velocity, stroke rate, and distance per stroke during elite swimming competition. Med. Sei. Sports Exerc. 17: 625-634.

Dengel, D.R., Flynn, M.G., Costill, D.L., and Kirwan, J.P. (1989). Determinants of success during triathlon competition. Res. Q. Exerc. Sport 60: 234-238.

Di Prampero, P.E. (1981). Energetics of muscular exercise. Rev. Physiol. Biochem. Pharmacol. 89: 143-223.

Farber, H.W., Schaefer, E.J., Franey, R., Grimaldi, R., and Hill, N.S. ( 1991). The endurance triathlon: Metabolic changes after each event and during recovery. Med. Sei. Sports Exerc. 23: 959-965.

Gnehm, P., Reichenbach, S., Altpeter, E., Widner, H., and Hoppeler, H. (1997). Influence of different racing positions on metabolic cost in elite cyclists. Med. Sei. Sports Exerc. 29: 818-823.

Hagan, R.D., Weis, S.E., and Raven, P.B. (1992). Effect of pedal rate on cardiorespiratory responses during continuous exercise. Med. Sei. Sports Exerc. 24: 1088-1095.

Hausswirth, C., Bigard, A.X., and Guézennec, C.Y. (1997). Relationships between running mechanics and energy cost of running at the end of a triathlon and a marathon. lnt. J. Sports Med. 18:330-339.

Hausswirth, C., and Lehénaff, D. (2001). Physiological demands of running during long distance runs and triathlons. Sports Med. 31:679-689.

Hausswirth, C., Lehénaff, D., Dréano, P., and Savonen, K. (1999). Effects of cycling alone or in a sheltered position on subsequent running performance during a triathlon. Med. Sei. Sports Exerc. 31 : 599-604. 
Howley, E.T., Basset, D.R., and Welch, H.G. (1995). Criteria for maximal oxygen uptake: Review and commentary. Med. Sei. Sports Exerc. 27: 1292-1301.

Hue, O., Le Gallais, D., Challet, D., Boussana, A., and Préfaut, C. (1998). The influence of prior cycling on biomechanical and cardiorespiratory response profiles during running in triathletes. Eur. J.Appt. Physiot. 77: 98-105.

Kreider, R.B., Boone, T., Thompson, W.R., Burkes, S., and Cortes, C.W. (1988). Cardiovascular and thermal responses of triathlon performance. Med. Sei. Sports Exerc. 20: 385-390.

Laursen, P.B., Rhodes, E.C., and Langill, R.H. (2000). The effects of 3000-m swimming on subsequent 3-h cycling performance: Implications for ultraendurance triathletes. Eur. J. Appt. Physiol. 83: 28-33.

Lavoie, J.M., and Montpetit, R.R. (1986). Applied physiology of swimming. Sports Med. 3: $165-189$.

Margaritis, 1. ( 1996). Facteurs timitants de la performance en triathlon. Can. J. Appt. Physiol. 21: $1-15$.

McLaughlin, J.E., King, G.A., Howley, E.T., Basset, D.R., Jr., and Ainsworth, B.E. (2001). Validation of the Cosmed K4 b2 portable metabolic system. Int. J. Sports Med. 22: 280-284.

Millet, G.P., and Vleck, V.E. (2000). Physiological and biomechanical adaptations to the cycle to run transition in Olympie triathlon: Review and practical recommendations for training. Br. J. Sports Med. 34: 384-390.

O'Toole, M.L., and Douglas, P.S. ( 1995). Applied physiology of a triathlon. Sports Med. 8: 201-225.

Pyne, D.B., Boston, T., Martin, D.T., and Logan, A. (2000). Evaluation of the Lactate Pro blood lactate analyser. Eur. J. Appt. Physiol. 82: 112-116.

Sargeant, A.J. (1994). Human power output and muscle fatigue. Int. J. Sports Med. 15: 116-121.

Schabort, E.J., Killian, S.C., Gibson, A.S., Hawley, J.A., and Noakes, T.D. (2000). Prediction of triathlon race time from laboratory testing in national triathletes. Med. Sei. Sports Exerc. 32: 844-849.

Toussaint, H.M., Bruinink, L., Coster, R., De Looze, M., Van Rossem, B., Van Veenen, R., and De Groot, G. ( 1989 ). Effect of a triathlon wet suit on drag during swimming. Med. Sei. Sports Exerc. 21: 325-328.

Trappe, T.A., Pease, D.L., Trappe, S.W., Troup, J.P., and Burke, E.R.(1996). Physiological responses to swimming while wearing a wet suit. Int. J. Sports Med. 17: 111-114.

Vercruyssen, F., Hausswirth, C., Smith, D., et Brisswalter, J. (2001). Effet de la durée de l'exercice sur le choix d'une cadence optimale de pédalage chez des triathlètes. Can. J. Appt. Physiol. 26: 44-54.

Wasserman, K., Whipp, B.J., Koyal, S.N., and Beaver, W.L. (1973). Anaerobic threshold and respiratory gas exchange during exercise. J. Appt Physiol. 35: 236-243.

Woledge, R.C. (1998). Possible effects of fatigue on muscle efficiency. Acta Physiol. Scand. 162: 267-273.

Zamparo, P., Capelli, C., Cautero, M., and Di Nino, A. (2000). Energy cost of front-crawl swimming at supra-maximal speeds and underwater torque in young swimmers. Eur. J.Appl. Physiol. 83: 487-491. 